\title{
Microstructure of NbTi superconducting alloy
}

\author{
M. B. Lazareva ${ }^{1}$, A. A. Mazilov ${ }^{2, \dagger}$, V. A. Ksenofontov ${ }^{1}$, T. I. Mazilova ${ }^{1}$, Ju. A. Gordienkoº \\ O. V. Dudka' I. M. Mikhailovskij ${ }^{1}$ \\ †alexey.mazilov@gmail.com
}

${ }^{1}$ NSC “Kharkov Institute of Physics and Technology”, Akademicheskaya Str., 1, Kharkov, 61108, Ukraine

${ }^{2}$ Belgorod National Research University, Pobedy Str., 85, Belgorod, 308015, Russia

\begin{abstract}
A superconducting multifilament wire made of $\mathrm{Nb}-60$ at.\% Ti alloy was investigated by methods of atom probe/field ion microscopy (APFIM), three-dimensional atom probe (3DAP), high resolution electron microscopy (HREM) and computer simulation. The results of the APFIM and HREM analysis suggest that the specimens contain two phases having bcc ( $\beta$-phase) and hcp ( $\alpha$-phase) structures. Mesoscopic and nanoscale heterogeneities of element concentration in $\beta$-phase were observed. Atom probe analyses of annealed $\mathrm{NbTi}$ superconductors and a statistical processing of the chains of atoms, consistently registered by mass-analyses, indicated that no atomic clusters were formed in the alloy prior to the onset of the second phase formation. The $\beta$-phase and niobium enriched phase have the configurations extended along the wire axis, the nanoscale heterogeneity inside the $\beta$-phases was nearly isotropic. An atomic structure of interphase boundaries has been determined using HREM and atomistic structure calculations. Interphase boundaries in Nb-Ti alloy are coherent or semi-coherent in spite of the existence of the irregular microprotrusions. The boundaries between $\alpha$ - and $\beta$-phases reveal the absence of the rigid-body translation and continuity of lattice planes across the interface. It was demonstrated that the method of simulation in reciprocal space is computationally efficient for investigations of the atomic structure and energy of coherent and noncoherent interphase boundaries. The extremely high critical current density in the optimized Nb-Ti can be due to the coherent structure of interphase boundaries.
\end{abstract}

Keywords: NbTi superconducting alloy, three-dimensional atom probe, high resolution electron microscopy, computer simulation, atomic structure, $\alpha$ - and $\beta$-phases.

\section{Introduction}

Superconductors are widely used due to their excellent advantages in the generation of intensive magnetic fields and large current transportation. In last decades, extremely high critical current densities have been reached by processing NbTi superconductors [1-4]. Superconductors of a high current-carrying capacity were obtained due to the formation of a heterophase composite structure during the decomposition of a supersaturated solid solution. There are several qualitative models describing the correlation between current-carrying capacity and change of a fine structure during treatment by plastic deformation and annealing [3-5]. Flux pinning in NbTi superconductors is usually described in connection with $\alpha$-Ti precipitates. There is good understanding of a pinning nature of these precipitates, but the role of a fine structure of a superconducting $\beta$-phase and normal conducting $\alpha$-Ti precipitates as flux pins have not been revealed until now. For this reason, it is necessary to explore the fine structure of the superconducting alloy, since it plays a critical role in various factors such as diffusion, segregation and decomposition, which have a strong effect on the current-carrying capacity. The objective of the present study is to characterize the atomic-level structure of the NbTi alloy, in particular to describe the atomic structure of boundaries between superconducting and normal conducting phases.

\section{Experimental procedure}

The niobium-titanium superconducting wire $(\mathrm{N}-48.5 \pm 1.5$ wt. $\% \mathrm{Ti}$ ) with diameter $1 \mathrm{~mm}$ was produced via the technique of electric arc melting at Ulba Metallurgical Plant. The main impurity content in the alloy did not exceed: $\mathrm{C}<0.04 ; \mathrm{N}<0.03$; $\mathrm{O}<0.05 ; \mathrm{Fe}<0.06$ wt.\%. The critical current density in a magnetic field with induction $5 \mathrm{~T}$ at $4.2 \mathrm{~K}$ was $1.8 \cdot 10^{5} \mathrm{~A} / \mathrm{cm}^{2}$. For an atomic level study of the local chemical structure of a high $-j_{c}$ NbTi alloy [6], the atom probe/field ion microscopy (APFIM) with energy compensation IONEX - VG 100 and the three-dimensional atom probe (3DAP) were used. Before manufacturing the tip samples for the field ion microscopy study the copper matrix was etched in a nitric acid and then electrochemical polishing of filaments in $\mathrm{HNO}_{3}(9)+\mathrm{HF}$ (1) solution was carried out. The pressure of residual gases in the atom probe during experiment was kept at a level of $10^{-10} \mathrm{~Pa}$; the pressure of the imaging gas, neon, was $10^{-3}-10^{-4} \mathrm{~Pa}$. The mass-analysis was carried out at $45-60 \mathrm{~K}$ and the amplitude of pulse voltage amounted $18-20 \%$ of the constant voltage for the best image. The effective diameter of the probe hole varied depending on the radius of curvature from 0.5 up 
to $1.0 \mathrm{~nm}$. Atomic level characterization of the alloy after annealing provided an information on the composition processes in this material.

The atomic structure of interphase boundaries has been determined using high resolution electron microscopy (HREM) and atomistic structure calculations. To image the structure of the interphase boundaries a high-resolution transmission electron microscope JEOL JEM-4000EX (II) was employed. Conventional electron microscopy methods such as the selected-area diffraction, bright-field and weakbeam techniques were used. The atomic relaxation near an interphase boundary was studied using a pair-potential analytic model [7,8]. This method of computation in a reciprocal space not only greatly reduces the number of variation parameters required in computation of an atomic relaxation but also leads to analytic expressions from which some general features of the grain boundary structure and thermodynamical properties can be deduced.

\section{Results and discussion}

\subsection{Heterogeneity of alloy microstructure}

The results of the transmission electron microscope (TEM) and HREM analysis suggest that the specimens contain two phases, body centered cubic (bcc) structure with a lattice parameter of $a=0.329 \mathrm{~nm}$ ( $\beta$-phase), and hexagonal closed packed (hcp) structure ( $\alpha$-phase). Fig. 1 shows a weak-beam TEM image (a) with electron microdiffraction pattern (b) of a specimen for the APFIM investigations. Electron beam is approximately parallel to $[111]_{b c c}$. In this study, heterogeneities of element concentration of two levels were observed in $\beta$-phase: a mesoscopic heterogeneity observable by mass-analyses during field evaporation of a sample to a depth of the order of $1 \mu \mathrm{m}$ and nanoscale fluctuations of elements concentration inside the $\beta$-phase.

The niobium concentration determined by averaging the mass-spectrometer data (Fig. 2) obtained when analyzing the $\beta$-phase with extension of $50-60 \mathrm{~nm}$, was changed in the process of evaporation to a depth of $1 \mu \mathrm{m}$ from 30 up to 65 at $\%$.

The heterogeneity of $\beta$-phase chemical structure essentially exceeded the alloy heterogeneity revealed by a comparison of the element concentration in various

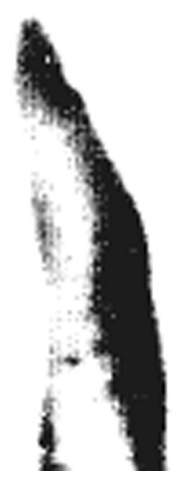

a

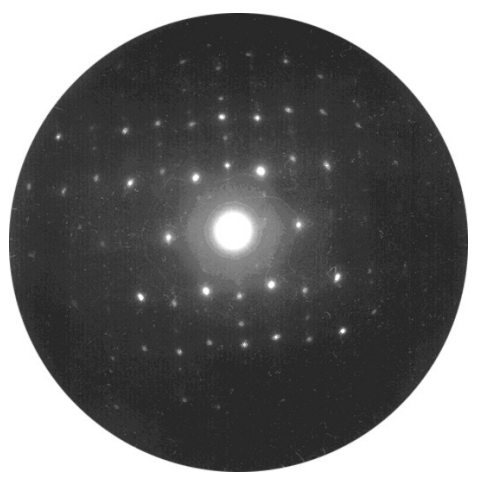

b
Fig. 1. A weak-beam TEM image (a) with electron microdiffraction pattern (b) of a needle-shape specimen oriented along the $[111]_{\mathrm{bcc}}$ direction. samples made from the superconducting wire of the same batch $( \pm 5$ at $\%)$. We observed also the fluctuations of a local element concentration inside the $\beta$-phase with characteristic linear sizes in an interval of 4-10 $\mathrm{nm}$. The statistically significant nanoscale heterogeneity of the structure inside such sites reached \pm 6 at. $\%$ and correlated with the observable peculiarities of the field emission contrast [6]. While the $\beta$-phase and niobium enriched phase have configurations extended along the wire axis, the nanoscale heterogeneity inside the $\beta$-phases observed in the present work was nearly isotropic.

One of possible reasons of element concentration variation inside the $\beta$-phase is a preferable clustering of titanium atoms, which can be considered as an initial stage of the $\beta$-phase precipitation. In this connection, the sequences of occurrence of $\mathrm{Ti}$ and $\mathrm{Nb}$ ions in the spectra of atom-by-atom field evaporation were considered and analyzed with the help of statistical methods. In these experiments, IONEX - VG 100 and needle-shaped specimens with the radius of $10-20 \mathrm{~nm}$ were used. FIM images of these tips were characterized by a high magnification and the effective size of the probe aperture was only slightly greater than the dimension of a single atom. Therefore, adjacent atoms in the sequence of detection were nearest or second-nearest neighbors in the specimen. A series of events on the atom-by-atom scale may be considered as a Markov chain, if the outcome of each event is dependent only on the previous event.

The titanium atoms distribution was investigated by examining the ion-by-ion APFIM data chain for $\mathrm{NbTiNb}$, $\mathrm{NbTiTiNb}$, NbTiTiTiNb, etc. sequences. If the chains of ions can be described as a random distribution, the probability of detecting a chain containing $n \mathrm{Ti}$ atoms can be expressed as $P(n)=p^{n} q^{2}$ and $D(n)=N P(n)$, were $p$ is probability of observing a Ti atom, $q=1-p$, and $N$ is the total number of atoms in the chain [9]. The significance of the experimental results is taken by $\left[D_{A P}(n)-D(n)\right] / \sigma$, where $D_{A P}(n)$ is the experimentally determined probability of detecting a chain containing $n \mathrm{Ti}$ atoms and $\sigma$ is given by $\sqrt{N p^{n} q^{2}}$. The significance was determined to be $-0.63,0.28,-0.629$ and -1.15 for $n=1,2,3$ and 4, correspondingly. Significances of the experimental values less then 2 and or greater than -2 indicate a random behavior.

Hence, at atomic level the ordering or formation of titanium atomic clusters in $\beta$-phase are not observed. The

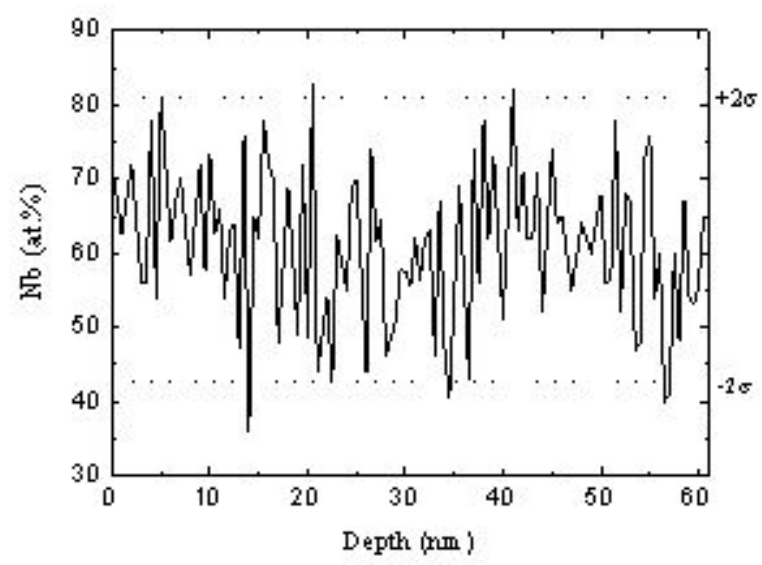

Fig. 2. Sections from the composition profile of $\mathrm{NbTi}$ alloy. 
last conclusion was confirmed by an analysis of FIM images and data obtained by using a three-dimensional atom probe (Fig. 3) in the process of field evaporation to a depth of the order of $10^{2} \mathrm{~nm}$.

These images also showed that in optimized high- $j$ $\mathrm{NbTi}$ superconductors no atomic clusters were formed homogeneously in a $\beta$-phase prior to the onset of the second phase formation. Such a finding was confirmed for $\beta$-phases with an elevated local concentration of titanium. In these regions, the registered chains of ions were also described as random statistical distributions. Our observations are consistent with extensive microstructural studies of $\mathrm{NbTi}$ alloys [1], which revealed that initially a Ti-rich $\alpha$-phase heterogeneously precipitated only at the grain boundaries and triple junctions in the $\beta$-solid solution.

\subsection{Atomic structure of interphase boundaries}

An analysis of the high resolution images and selected-area diffraction patterns showed that the orientation relationship between bcc and hcp phases was in all cases of the Burgers relation without any systematic deviations. This relation is characterized by parallelism of a pair of close-packed planes $(110)_{b c c}$ and $(0001)_{h c p}$, and pair of common close-packed directions contained within them. The interplanar spacing of two sets of the parallel planes in both crystals evaluated by Fourier transformation analysis of the HREM images was $0.233 \mathrm{~nm}$. The selected area diffraction patterns pointed out a fairly uniform distribution of grain orientation around $\langle 110\rangle_{\text {bcc }}$ drawing direction. In the HREM experiments the atomic columns were located at the maximum of the intensity in the major parts of the usable specimen thickness range. The atomic columns appear white under these experimental conditions so that the atomic structure in the vicinity of the grain boundaries was unambiguously resolved. A HREM image of the boundary between bcc and hcp phases acquired parallel to the $[111]_{\mathrm{bcc}} / /[2 \overline{1} \overline{1} 0]_{\mathrm{hcp}}$ axis is shown in Fig. 4. The upper portion of the image is $\beta$-phase and the lower is $\alpha$-phase. Observations of the boundary between $\alpha$ - and $\beta$-phases identify the crystalline nature of the interface. Analysis of the morphology of the interphase boundaries showed that the interfaces at the microscopic level are rough. Irregular microprotrusions with irrational orientations 1-3 nm high were observed. As a result in the HREM

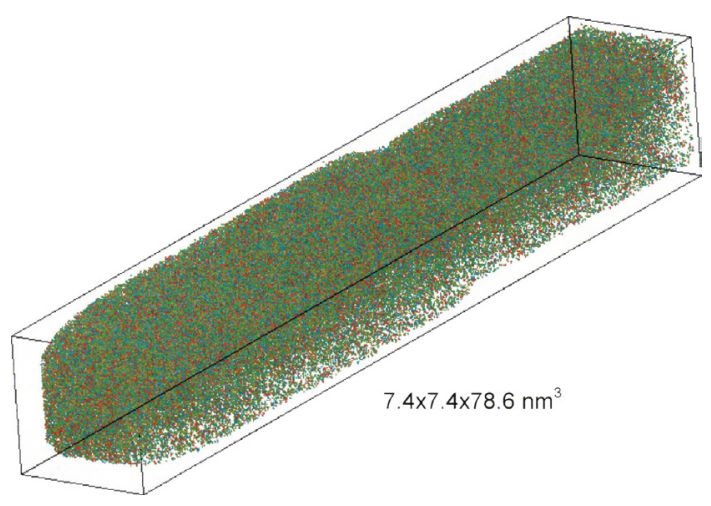

Fig. 3. (Color online) 3DAP elemental mapping of $\mathrm{Nb}-60$ at. \% $\mathrm{Ti}$ alloy. Red, green and blue dots are titanium, niobium and oxygen atoms respectively. experiments the planar sections of the interface oriented along an electron beam, only occasionally were observed. In most cases the superposition of the atomic columns of bcc and hcp phases took place in the core boundary regions. It was accompanied by an artificial spreading of the boundary core and relatively high noise level in the interface image.

The information limit of this microscope is approximately $0.14 \mathrm{~nm}$. Thus only three $(110)_{\mathrm{bcc}}$ and one $(0001)_{\mathrm{hcp}}$ sets of planes are resolved as fringes in the image. Careful inspection of the high-resolution image of Fig. 4 reveals the absence of the rigid-body translation of adjacent grains and demonstrates continuity of lattice planes across the interface. Moreover, almost no internal strain field contrast was observed in the interphase boundaries. Therefore, we can conclude that interphase boundaries in $\mathrm{Nb}$ - $\mathrm{Ti}$ alloy are coherent or semi-coherent in spite of the existence of the irregular microprotrusions.

In order to arrive at a description of an interphase boundary structure, an essential factor that needs to be determined is the state of rigid-body shift of neighboring grains and crystallographic planes in vicinity of the boundary core. An atomic configuration of the cores can be adequately described by both the lattice displacements for adjacent crystallites and rigid displacement of near-boundary atomic layers [7].

In analytical treatment in a reciprocal-space formulation, we used an effective pair potential for hcp metals [10] based on fitting to perfect crystal lattice properties. To obtain an analytical expression for the energy of an interface, the effective pair potential was used in the form

$$
v(r)=\sum_{p} D_{p} \exp \left(-\beta_{p} r\right)
$$

where $r$ is the distance between atoms, $D_{p}$ and $\beta_{p}$ are adjustable parameters. Usually most of the interphase boundary energy in alloys is attributed to the structural difference across the boundary, and the chemical contribution to the energy is small [11]. Taking into account this fact, a simulation block is assumed to be pure titanium. The potential parameters for $\mathrm{Ti}$ are: $D_{p}=769.04,-736.23,217.81$ and $-3.5434 \mathrm{eV}, \beta_{p}=1.6485$, $1.3552,1.1290$ and $0.66480 \AA^{-1}$ for $\mathrm{p}=0,1,2$ and 3 respectively.

The energy of a bicrystal is represented by a sum of interactions of atoms in $j$ and $k$ atomic layers. The interaction energy of all atomic layers per unit area may be expressed as follows [8]:

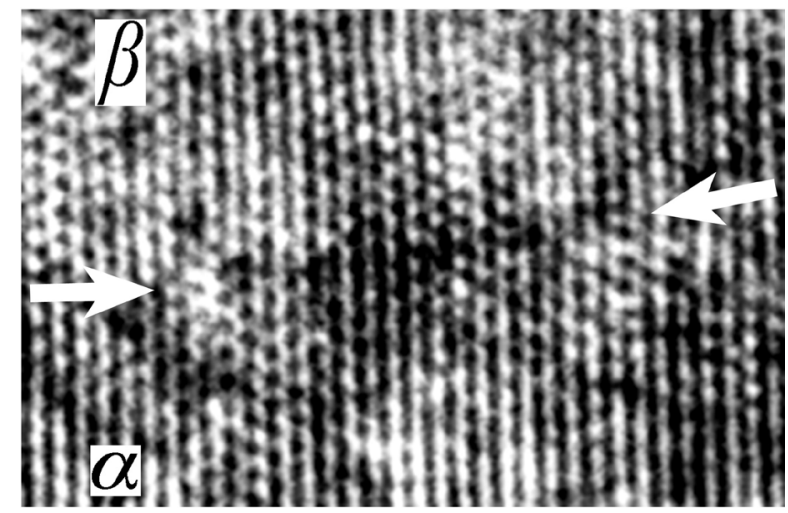

Fig. 4. High resolution transmission electron micrograph of the interphase boundary in NbTi alloy (shown by arrows). The rigidbody translation and misfit dislocations are absent. 


$$
\begin{aligned}
W & =\pi \sigma^{2} \sum_{j} \sum_{k \neq j} \sum_{l m} \sum_{p} \frac{\beta_{p} D_{p}}{q_{p l m}^{3}}\left(1+q_{p l m}\left|z_{j k}\right|\right) \times \\
& \times \exp \left(-q_{p l m}\left|z_{j k}\right|\right) \cos \left(g_{x l} T_{x j k}\right) \cos \left(g_{y m} T_{y j k}\right),
\end{aligned}
$$

Here $\sigma$ is the planar atomic density, $z_{j k}$ is the separation between the lattices, $\vec{T}_{j k}$ denote an arbitrary relative translation, $g_{x l}=2 \pi l / a_{x}$ and $g_{y m}=2 \pi m / a_{y}$, where $a_{x}$ and $a_{y}$ are the lattice periods in the $x$ and $y$ directions, $l, m$ are summation indices locating points in the planar reciprocal lattice, and $q_{p l m}=\sqrt{\beta_{p}^{2}+g_{x l}^{2}+g_{y m}^{2}}$.

In our calculations the positions of atomic layers were labelled by integers $i, j=-15 \ldots+15$. The wave-vector numbers were in the interval $|l, m| \leq 20$, but only those terms referring to near-surface planes and having both $|l| \leq 4$ and $|m| \leq 4$ contribute significantly to the sum in $W$. The first stage of relaxation was rigid-body shift of the adjacent crystals. After that plane-by-plane relaxation was done in both grains. The interphase boundary energy was obtained by subtracting the internal energy of perfect bcc and hcp crystals from the energy of the simulation block.

The predicted minimum energy structure for a coherent boundary between the $\alpha$ - and $\beta$-phases in Ti is shown in Fig. 5. The boundary has common axis $[111]_{\mathrm{bcc}} / /[2 \overline{1} \overline{1} 0]_{\mathrm{hcp}}$ and is viewed along the $[110]_{\mathrm{bcc}} / /[0001]_{\mathrm{hcp}}$ direction. The lowest energy corresponds to contraction of bcc crystal perpendicular to the boundary of $0.279 \AA$ and the rigid body shift along the [111] direction of $0.410 \AA$. As a result of relaxation, periodic rows of the tetrahedral structure units were formed in a core of the boundary. The rigidbody shift along the $[110]_{\mathrm{bcc}} / /[0001]_{\mathrm{hcp}}$ direction was absent. Corresponding close-packed planes in bcc and hcp phases were in exact registry at the interface. The interphase energy for a coherent boundary obtained in computer experiments was $90.41 \mathrm{~mJ} / \mathrm{m}^{2}$.

Non-coherent interphase boundary was produced by a rotation of the bcc grain about the $[211]_{\mathrm{bcc}}$ direction. In term of the analytical model of the interface $[6,7]$ an incommensurate non-coherent boundary can be obtained by a reducing the sets $\left\{\vec{G}^{j}\right\}$ and $\left\{\vec{G}^{k}\right\}$ to $G^{j}=G^{k}=0(l=m=0)$. In this case the violation of the Burgers orientation relationship between bcc and hcp phases takes place. Such a boundary has irrational

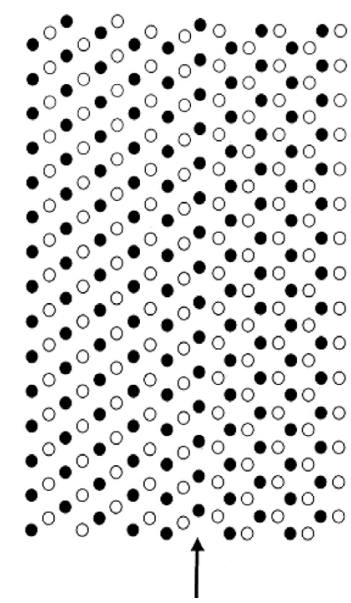

Fig. 5. Structure of the coherent interphase boundary predicted by atomistic simulation in reciprocal space. orientation relationships with respect to the adjacent matrix grain. The obtained energy of the incommensurate boundary is $992 \mathrm{~mJ} / \mathrm{m}^{2}$. This value is considerably higher than the energy of the coherent interphase boundary, and only slightly differs from the energy of a non-coherent high-angle grain boundary in $\mathrm{Ti}\left(758 \mathrm{~mJ} / \mathrm{m}^{2}\right.$ [12]). Perhaps, a high value of the energy of incommensurate interphase boundaries is responsible for the absence in NbTi alloy of interfaces with violation of the Burgers orientation relationships.

The universal edge-to-edge matching model $[13,14]$ was proposed to predict the orientation relationships between the two phases formed by a diffusional transformation. According to this model, a directional matching of atoms on the corresponding close-packed planes is a decisive factor that controls the orientation relationships between the two phases. In the present $\mathrm{Nb}$-Ti system, the parallelism is satisfied between the close-packed planes of the two phases, $(110)_{\mathrm{bcc}} / /(0001)_{\mathrm{hcp}}$, and the atomic row matching along closepacked directions, $[111]_{\mathrm{bcc}} / /[2 \overline{1} \overline{1} 0]_{\mathrm{hcp}}$ is observed. Hence, the observed orientation relationship is rationalized on the basis of the directional atomic matching on the close-packed planes along the close-packed directions. A flux pinning by a network of interphase boundaries can result in high critical current densities in superconductors [1,2]. The coherent interphase boundary structures observed in the optimized NbTi alloy, can be responsible for a strong flux pinning which is not accompanied by lattice distortions and thereby do not result in a significant degradation of superconducting properties.

\section{Conclusion}

A superconducting multifilament wire made of an optimized high- $j \mathrm{Nb}-60$ at.\% Ti alloy was investigated by APFIM, 3DAP, TEM, HREM and computer simulation. The results of the TEM and HREM analysis suggest, that the specimens contain two phases, a bcc structure with a lattice parameter of $a=0.329 \mathrm{~nm}$ ( $\beta$-phase), and a hcp structure ( $\alpha$-phase). APFIM and 3DAP researches revealed the mesoscopic and nanoscale heterogeneities of element concentration in $\beta$-phase. While the $\beta$-phase and niobium enriched phase have the configurations extended along the wire axis, the nanoscale heterogeneity inside the $\beta$-phases was nearly isotropic. FIM and 3DAP observations and a statistical processing of the chains of atoms, consistently registered by mass-analyses, showed that at atomic level the orderings or formations of titanium atomic clusters in the $\beta$-phase are not observed. This finding is consistent with previous TEM studies of NbTi alloys revealed that a Ti-rich $\alpha$-phase heterogeneously precipitated at the grain boundaries in the $\beta$-solid solution.

It was demonstrated that the method of simulation in reciprocal space is computationally efficient for investigations of the atomic structure and energy of coherent and noncoherent interphase boundaries. HREM investigations revealed coherent interfaces with the perfect registry of atomic planes and the absence of rigid-body translations of adjacent grains. In the optimized $\mathrm{Nb}$ - $\mathrm{Ti}$, the extremely high critical current density can be due to the coherent structure of interphase boundaries. 
Acknowledgements. We are grateful to Dr. N. Wanderka for insightful comments and support with the 3DAP and HREM analysis. The Russian Science Foundation (project $N$ 15-12-10019) supported this work.

\section{References}

1. D. A.Gardwell, D. S. Ginley. Handbook of superconducting materials. Superconductivity, materials and processes. London, IOP Publishing. (2003) 1075 p.

2. O.I. Volchok, M. B. Lazareva, A. V. Mats, Ya. D. Starodubov, N. A. Chernyak, O. V. Chernyi. Low Temp. Phys. 31, 894 (2005).

3. C. Bormio-Nunes, M. J. R Sandim, L. Ghivelder. J. Phys.: Condens. Matter. 19, 446204 (2007).

4. V.A. Beloshenko, V. V. Chishko. Phys. Met. Metallogr. 114 (12), 992 (2013).

5. G. E. Storozhilov, N. F. Andrievskaya, M. A. Tikhonovskii.
Phys. Met. Metallogr. 115 (7), 710 (2014).

6. B.G. Lazarev, V.A. Ksenofontov, I.M. Mikhailovskij, O. A. Velikodnaya. Low Temp. Phys. 24, 205 (1998).

7. T.I. Mazilova, V.A. Ksenofontov, V.N. Voyevodin, E. V. Sadanov, I. M. Mikhailovskij. Phil. Mag. Lett. 91, 304 (2011).

8. I.M. Mikhailovskij, T.I. Mazilova, V.N. Voyevodin, A. A. Mazilov. Phys. Rev. B. 83, 134115 (2011).

9. M.K. Miller, A. Cerezo, M.G. Hetherington, G. D. W. Smith. Atom-probe field ion microscopy. Oxford, Oxford University Press. (1996) 509 p.

10. R. Pasianot, E. J. Savino. Phys. Rev. B. 45, 12704 (1992).

11. J. K. Chen, D. Farkas, W.T. Reynolds Jr. Acta Mater. 45, 4415 (1997).

12. Y. C. Wang, H. Q. Ye. Phil. Mag. A. 75, 261 (1997).

13. M.X. Zhang, P. M. Kelly. Scr. Mater. 52, 963 (2005).

14. A.S. Bakaŭ, I.M. Mikhailovskij, T.I. Mazilova, N. Wanderka. Low Temp. Phys. 28, 279 (2002). 